\title{
Spontaneous Spinal Epidural Hematoma: A Retrospective Study on Prognostic Factors and Review of the Literature
}

\author{
Mark Fedor ${ }^{1}$, Eric S. Kim ${ }^{1}$ Kai Ding ${ }^{2}$, J. Paul Muizelaar', Kee D. Kim ${ }^{1}$ \\ ${ }^{I}$ Department of Neurological Surgery, University of California, Davis Medical Center \\ ${ }^{2}$ Division of Biostatistics, Department of Public Health Sciences, University of California, Davis
}

\begin{abstract}
Objectives: The spontaneous spinal epidural hematoma (SSEH) is a rare clinical entity. Patients typically present with sudden onset back pain followed by neurological deficits.

Methods: Diagnosis of SSEH is usually made with MRI and standard treatment is surgical evacuation. In 1996, Groen published the most comprehensive review on the SSEH in which he analyzed 333 cases. We review 104 cases of SSEH presented in the English literature since the last major review and add three of our own cases, for a total of 107 cases. Results: Our patients presented with back pain and neurologic deficits. Two made excellent functional recovery with prompt surgical decompression while one continued to have significant deficits despite evacuation. Better postoperative outcome was associated with less initial neurological dysfunction, shorter time to operation from symptom onset and male patients. Conclusion: We discuss the etiology of SSEH and report current trends in diagnosis, treatment, and outcome.
\end{abstract}

Key Words: Spinal epidural hematoma $\cdot$ Back pain $\cdot$ Neurological deficits $\cdot$ MRI

\section{INTRODUCTION}

\section{Case 1}

\section{1) History}

The patient is a 71 year-old right handed man who presented to the emergency room with sudden onset of upper back pain, just below his neck, associated with right arm weakness. Within hours, he developed weakness of his legs associated with paresthesia that progressed to paralysis of bilateral lower extremities. Past medical history is significant for arthritis, restless leg syndrome diagnosed six days prior to presentation, and history of syncope.

\section{2) Examination}

He had $4+/ 5$ strength in the left upper extremity and $4 / 5$

- Received: November 18, 2011 • Revised: December 20, 2011

- Accepted: January 5, 2012

Corresponding Author: Kee D. Kim, MD

Department of Neurological Surgery, University of California Davis

School of Medicine, 4860 Y St. Suite 3740, Sacramento, CA 95817

Tel: +916-734-3102, Fax: +916-703-5368

Email: kdkim@ucdavis.edu strength in the right upper extremity with complete paralysis of bilateral lower extremities. Although sensation to light touch was preserved, he had decreased pin-prick sensation and proprioception starting at the cervicothoracic level, worse on the right side than the left. Deep tendon reflexes were brisk and the Babinski reflexes were up-going. He had normal coagulation values.

\section{3) Imaging}

MRI showed large posterolateral extradural mass extending from C2 to T4 with marked spinal cord compression. T1weighted images showed low signal intensities and T2-weighted images showed heterogeneous intermediate to high signal intensities. No contrast enhancement was found except for a small nodular enhancing focus at the C7-T1 junction to the right of the midline (Fig. 1).

\section{4) Operation}

The patient was taken urgently to the operating room for a C3 to T1 laminectomy. A dark blood clot was noted in the epidural space and was evacuated. Portions of the epidural clot were sent to pathology whose report confirmed the presence of hematoma with no evidence of vascular malformation. 


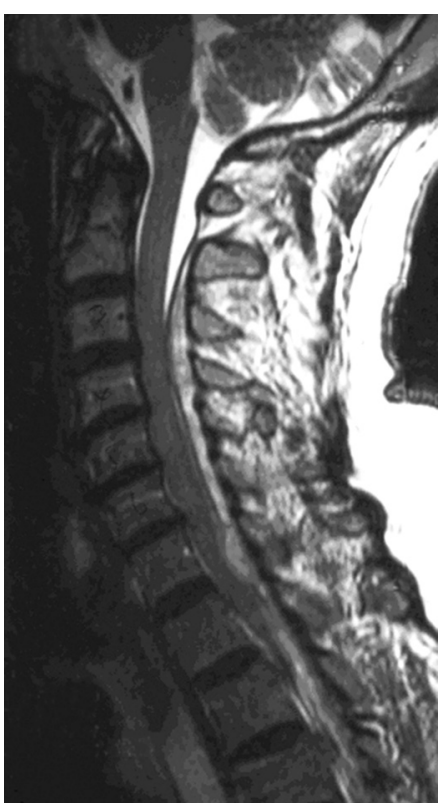

Fig. 1. T2-weighted sagittal magnetic resonance (MR) image showing large posterolateral extradural mass extending from C2 to T4 with marked spinal cord compression.

\section{5) Postoperative Course}

Postoperative MRI showed complete resolution of the hematoma (Fig. 2). The patient recovered well with prompt return of neurologic function. On two-year follow up, he was doing well with no residual neurological deficits, only occasional spasms in his trapezius muscle.

\section{Case 2}

\section{1) History}

Patient is a 78 year-old woman who awoke early in the morning with low back pain. Soon afterwards, she presented to the emergency room with additional complaint of right lower extremity pain and weakness with difficulty ambulating. Her past medical history was significant for hypertension, type II diabetes and distant history of TB, previously treated. She had normal coagulation values.

\section{2) Examination}

On neurological examination, she had 4/5 right dorsiflexion weakness but otherwise had good motor strength. She had diminished sensation to light touch in her right lower extremity.

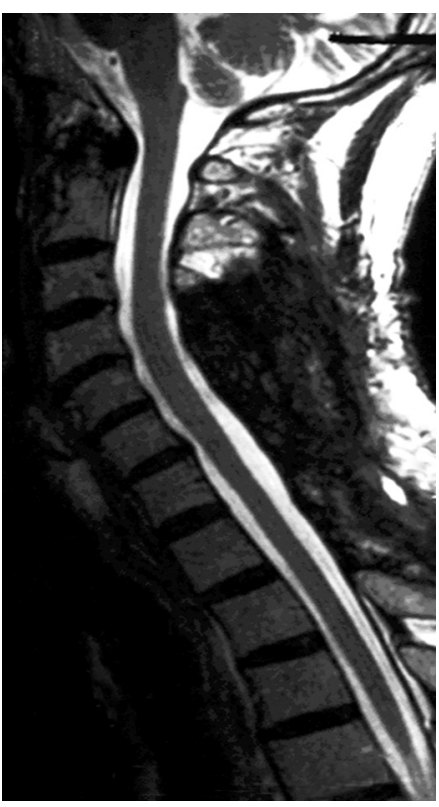

Fig. 2. Postoperative magnetic resonance image shows complete resolution of the hematoma.

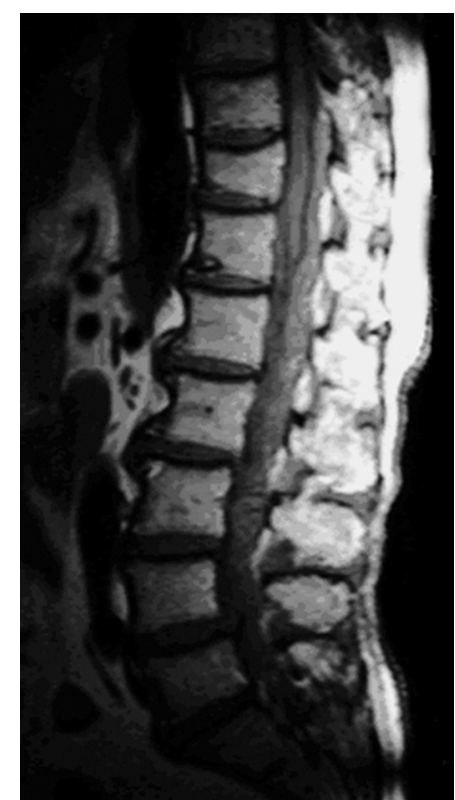

Fig. 3. T1-weighted sagittal MR image showing epidural mass from $\mathrm{T} 10$ to $L 4$

\section{3) Imaging}

Initial MRI showed epidural mass from T10 to L4 (Fig. 3).

\section{4) Operation}

Patient was taken to the operating room 16 hours after 


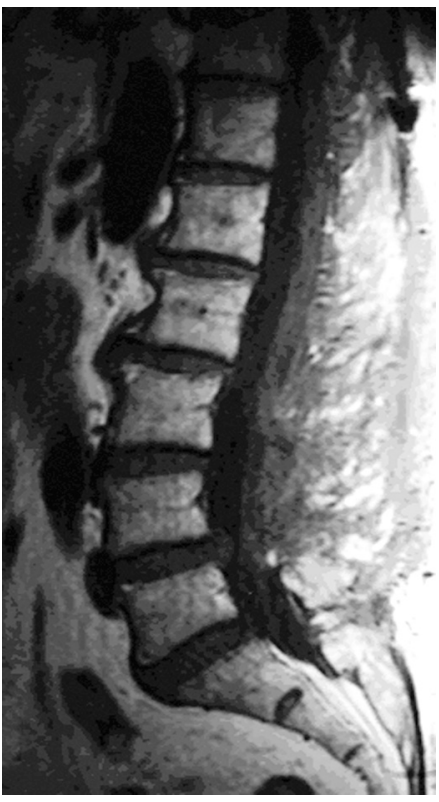

Fig. 4. Postoperative MR image revealed complete resolution of the hematoma.

the initial symptom of low back pain. She underwent T10 to L4 laminectomy and was noted to have significant compression of the thecal sac from epidural blood clot. The hematoma was evacuated and portions of the specimen were sent to pathology, where findings were consistent with hematoma with no evidence of vascular malformation.

\section{5) Postoperative Course}

Postoperative MRI showed complete resolution of the hematoma (Fig. 4). Patient showed progressive improvement and eventually gained return of full strength in her right leg. She however, continued to have right leg pain and numbness

\section{Case 3}

\section{1) History}

A 42 year old woman was gardening when she suddenly developed severe low back pain with nausea. The day prior she had been engaged in heavy physical work. Soon after lying down she noticed she could not move her legs and could not feel anything from the waist down. Past medical history is significant for Aspirin use.

\section{2) Examination}

Both legs were completely flaccid. There were absent re-

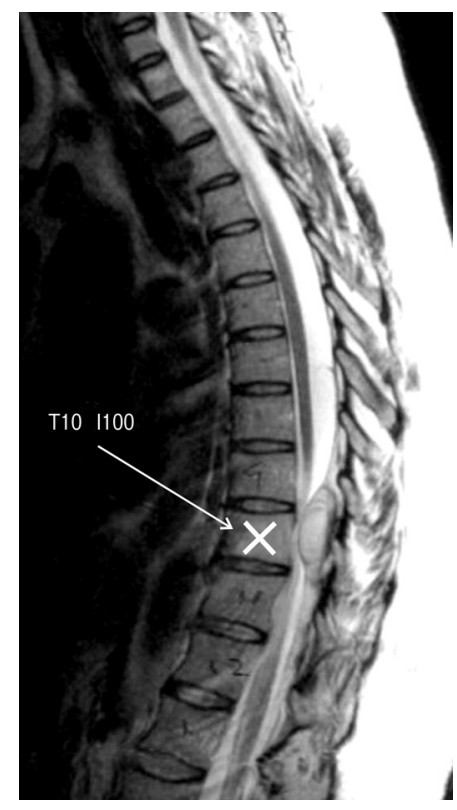

Fig. 5. T2-weighted sagittal MR image shows an extradural mass compressing the spinal cord from T9 to T11.

flexes in her legs. She also could not feel anything below the T11 sensory level. She had urinary retention as well.

\section{3) Imaging}

MRI showed an extradural mass compressing the spinal cord from T9 to T11. It was hyperintense to the spinal cord on T2 imaging. The signal was heterogeneous in character (Fig. 5).

\section{4) Operation}

She had a T9 to L1 laminectomy performed within 24 hours of symptom onset. The hematoma was found to extend down to L1 intraoperatively. Pathological analysis revealed only blood clot.

\section{5) Postoperative Course}

The patient had no improvement. She remained paraplegic and has made little improvement with rehabilitation.

\section{MATERIALS AND METHODS}

The last major review on SSEH was reported by Groen in $1996^{41)}$. Therefore, our literature search was limited to cases reported since 1996 coinciding with the widespread acceptance and use of MRI. A PubMed literature search for "spon- 
taneous spinal epidural hematoma" was conducted and the reports from the English literature were collected. Hematomas associated with vascular malformations, spinal surgery, trauma, spinal tumors, or any spinal procedures such as diagnostic lumbar punctures were excluded. Patients on anti-coagulation medication and those with hypertension were included since they were part of the past reviews on SSEH. The diagnosis was made with an imaging study and confirmed in most cases by surgery.

We statistically investigated the effect of time to surgery (before or after 24 hours of hospital admission) on neurological outcome. Because the literature accounts were inconsistent in their clinical descriptions of initial and final neurological status, the Frankel grading system was used to provide a standardized assessment of neurological dysfunction. The initial and final Frankel grade for each patient was assigned based on symptomatic reports (Table 1). For quantitative analysis, the Frankel grade scale of A-E was converted to a 1-5 scale, respectively. We used linear regression to investigate the relationship between final Frankel grade and time to surgery. Initial Frankel grade, age, sex, time to surgery, size of

Table 1. Frankel grade system

\begin{tabular}{cl}
\hline \hline $\begin{array}{c}\text { Frankel } \\
\text { Grade }\end{array}$ & Description \\
\hline A & Complete motor and sensory loss \\
B & Complete motor loss and incomplete sensory loss \\
C & Incomplete motor loss, with "useless" or non-functio- \\
& nal strength \\
D & Incomplete motor loss, with "useful" or functional \\
& strength \\
E & No motor or sensory abnormalities \\
\hline
\end{tabular}

hematoma, history of hypertension, and use of anticoagulants were included as covariates. We first considered the effects of time to surgery, initial Frankel grade, age, and sex and then sequentially added two-way interactions as well as effects associated with size of hematoma, hypertension and use of anticoagulants. Four age categories were used: less than 20 years, 20 to 40 years, 40 to 60 years and 60 years of age and older. Statistical analyses were conducted using $\mathrm{R}$ version 2.12.1 and a significance level of 0.05 was used to determine significant effects.

\section{RESULTS}

We identified 104 cases of SSEH in the literature and added our three patients for a total of 107 cases (Table 2). Nineteen patients had no significant past medical history. Fourteen patients had a history of anticoagulation (including use of Aspirin and Ibuprofen) and thirteen had a history of hypertension and five patients had hemophilia. Other past medical histories of significance included previous stroke, pregnancy, amyloidosis, renal transplant, epilepsy, and a prior history of SSEH. In thirteen patients the past medical history was not presented. The neurological deficits varied in severity from minor sensory abnormalities to complete paralysis. In four patients, severe neck pain or headache was the only presenting symptom. The majority of patients $(93 / 107)$ presented with neurological impairment leading to loss of function (Table 3).

In 98 cases, MRI was used to make the diagnosis of SSEH. A CT scan was performed in five and a CT myelogram was performed in three of the cases. A conventional myelogram was used in one case. The average number of spinal segments

\section{SSEH Distribution by Spinal Level}

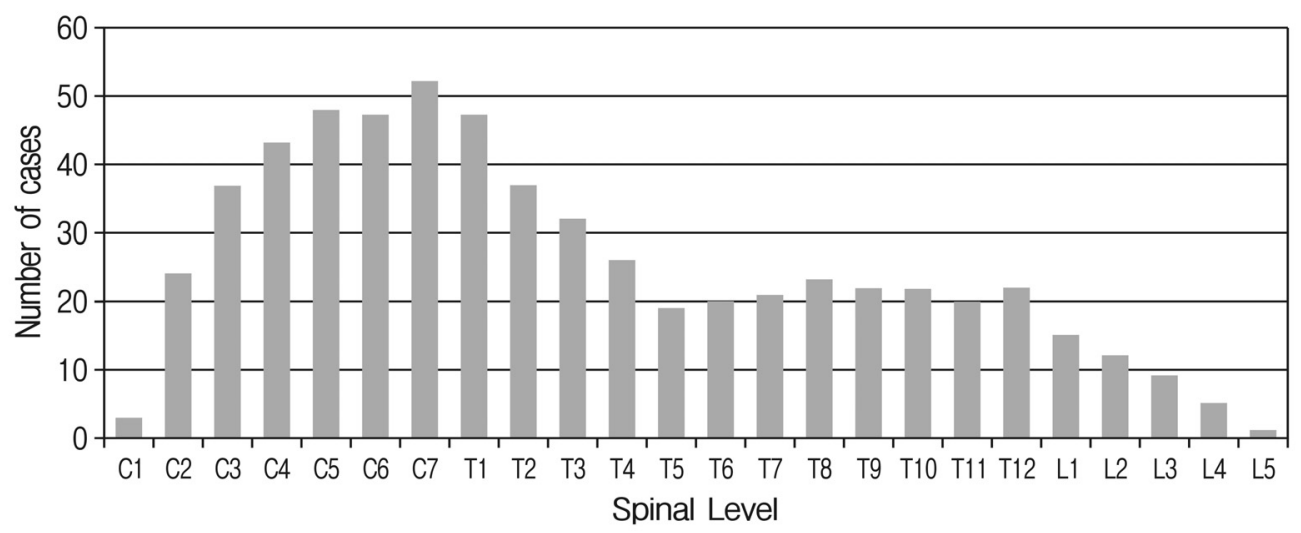

Fig. 6. Distribution of spinal segments involved in all reviewed cases of SSEH. There is increased frequency in the cenvico-thoracic and to a less degree, the thoraco-lumbar regions. Overall there is increased involvement of the cervical and upper thoracic regions. 
Table 2. Demographics of SSEH patients

\begin{tabular}{|c|c|c|c|c|c|c|c|c|c|c|}
\hline Author & Year & Age & Gender & Level & Position & $\begin{array}{l}\text { Initial } \\
\text { Frankel }\end{array}$ & $\begin{array}{c}\text { Time to } \\
\text { Operation (hrs) }\end{array}$ & $\begin{array}{l}\text { Final } \\
\text { Frankel }\end{array}$ & $\begin{array}{l}\text { Duration of } \\
\text { Symptoms }\end{array}$ & PMH \\
\hline Alabedeen $^{21}$ & 1996 & 79 & $M$ & T6-T8 & $P$ & $\mathrm{D}$ & $>48$ & $E$ & hrs & None \\
\hline Wagner ${ }^{3)}$ & 1996 & 74 & $M$ & C2-C6 & $\mathrm{PL}$ & A & No OR & E & $\min$ & spasticity \\
\hline Silber ${ }^{4)}$ & 1996 & 51 & $M$ & C5-T4 & $P L$ & A & No OR & $E$ & $2 \mathrm{~h}$ & Ibuprofen \\
\hline Chen $^{5)}$ & 1997 & 50 & $M$ & $\mathrm{~T} 4$ & $A$ & A & $<24$ & C & $\min$ & None \\
\hline Kotilainen ${ }^{6)}$ & 1997 & 13 & M & L4-L5 & $P L$ & D & $<24$ & $E$ & $\min$ & None \\
\hline Lonjon $^{7)}$ & 1997 & 61 & $M$ & $\mathrm{C} 5-\mathrm{C} 7$ & $P L$ & D & $<24$ & $E$ & $\min$ & None \\
\hline (arroll| & 1997 & 26 & $\mathrm{~F}$ & T5-T8 & A & C & $<24$ & D & $\min$ & pregnant \\
\hline Lonjon ${ }^{7)}$ & 1997 & 73 & M & L3-L4 & P & C & $>48$ & C & lwk & None \\
\hline Lonjon ${ }^{7)}$ & 1997 & 76 & $\mathrm{~F}$ & C2-C5 & A & A & $>48$ & C & $\min$ & AntiCoag \\
\hline David $^{9)}$ & 1997 & 23 & $M$ & T4-T9 & $P L$ & A & No OR & $\mathrm{E}$ & $1 d$ & None \\
\hline Alexiadou ${ }^{10)}$ & 1998 & 66 & M & T12-L1 & $P$ & A & $<12$ & D & Unk & AntiCoag \\
\hline Pecha ${ }^{11)}$ & 1998 & 13 & M & T1-T4 & $P$ & A & 8 & $\mathrm{E}$ & $2 d$ & None \\
\hline Schaeybroeck ${ }^{12)}$ & 1998 & 75 & $\mathrm{~F}$ & T1-T5 & $P$ & A & 18 & D & $18 \mathrm{~h}$ & TPA \\
\hline Awada ${ }^{13)}$ & 1998 & 14 & $M$ & C2-T2 & A & A & 9 & C & $\min$ & seizure \\
\hline Henderson ${ }^{14)}$ & 1998 & 77 & $\mathrm{~F}$ & T6-L3 & A & A & 11 & D & $\min$ & None \\
\hline Alexiadou $^{10)}$ & 1998 & 51 & M & $\mathrm{T} 12$ & P & C & $>48$ & $\mathrm{E}$ & Unk & AntiCoag \\
\hline Schaeybroeck ${ }^{12)}$ & 1998 & 46 & M & T11-T12 & P & C & $>48$ & $\mathrm{E}$ & $3 d$ & AntiCoag \\
\hline Meena $^{15)}$ & 1998 & 26 & M & C5-T1 & P & C & $>48$ & $\mathrm{D}$ & $15 d$ & Hemophelia B \\
\hline Patel $^{16)}$ & 1998 & 1.83 & $\mathrm{~F}$ & C7-T10 & $P$ & C & $>48$ & $\mathrm{E}$ & $6 d$ & None \\
\hline Alexiadou ${ }^{10)}$ & 1998 & 71 & $\mathrm{~F}$ & $\mathrm{~T} 12-\mathrm{L} 1$ & $P$ & A & $>12$ & A & Unk & alcohol \\
\hline Alexiadou ${ }^{10)}$ & 1998 & 87 & $M$ & C5-T3 & $P$ & A & $>12$ & A & Unk & AntiCoag \\
\hline Patel $^{16)}$ & 1998 & 1.5 & M & Cl-T1 & $P$ & A & 24 & D & $5 d$ & None \\
\hline Pahapill $^{17)}$ & 1998 & 30 & M & T3-T4 & $\mathrm{PL}$ & D & No OR & $\mathrm{E}$ & hrs & None \\
\hline Fukui $^{18)}$ & 1999 & 67 & M & C3-C5 & $\mathrm{PL}$ & C & 10 & D & Unk & Unk \\
\hline Fukui $^{24)}$ & 1999 & 73 & $M$ & T6-L3 & $P$ & C & 23 & D & Unk & Unk \\
\hline Fukui $^{24)}$ & 1999 & 40 & M & L3-L4 & $A P$ & A & 50 & D & Unk & Unk \\
\hline Cirak $^{19)}$ & 1999 & 8 & M & C3-C7 & A & A & $>48$ & $\mathrm{E}$ & $9 d$ & None \\
\hline Fukui ${ }^{24)}$ & 1999 & 81 & $\mathrm{~F}$ & T7-L3 & $P$ & C & 26 & $E$ & Unk & Unk \\
\hline Fukui $^{24)}$ & 1999 & 31 & $\mathrm{~F}$ & $\mathrm{Cl}-\mathrm{C} 3$ & $\mathrm{AL}$ & $\mathrm{E}$ & No OR & $E$ & Unk & Unk \\
\hline Fukui $^{24)}$ & 1999 & 54 & $\mathrm{~F}$ & C6-T1 & $A$ & D & No OR & $E$ & Unk & Unk \\
\hline Fukui $^{24)}$ & 1999 & 76 & M & $\mathrm{C} 2-\mathrm{C} 6$ & $P$ & $E$ & No OR & $E$ & Unk & Unk \\
\hline Fukui $^{24)}$ & 1999 & 42 & M & C2-T6 & $P$ & E & No OR & $\mathrm{E}$ & Unk & Unk \\
\hline Gaffney $^{20)}$ & 2000 & 70 & M & C3 & $P L$ & D & $<24$ & $\mathrm{E}$ & Unk & HTN \\
\hline$v$ Heesewijik ${ }^{21)}$ & 2000 & 4 & $\mathrm{~F}$ & $\mathrm{C} 2-\mathrm{C} 6$ & $\mathrm{PL}$ & C & $<24$ & $\mathrm{E}$ & $15 d$ & None \\
\hline Inamasu ${ }^{22)}$ & 2000 & 50 & $M$ & C3-C6 & $P$ & C & No OR & E & $4 \mathrm{~h}$ & None \\
\hline Borges $^{23)}$ & 2000 & 74 & $M$ & $\mathrm{C} 4-\mathrm{T} 1$ & $P L$ & A & No OR & $\mathrm{E}$ & hrs & AntiCoag \\
\hline Duffill $^{24)}$ & 2000 & 64 & $\mathrm{~F}$ & C2-T1 & $P$ & A & No OR & $\mathrm{E}$ & $30 \mathrm{~min}$ & None \\
\hline Duffill $^{24)}$ & 2000 & 28 & $M$ & C7-T6 & P & C & No OR & $\mathrm{E}$ & $\min$ & None \\
\hline Duffill $^{24)}$ & 2000 & 72 & M & C2-T2 & $\mathrm{PL}$ & C & No OR & $\mathrm{E}$ & hrs & None \\
\hline Duffill $^{24)}$ & 2000 & 68 & $\mathrm{~F}$ & T4-T1 1 & $\mathrm{P}$ & A & No OR & $\mathrm{E}$ & $\min$ & HTN \\
\hline Hentschel $^{25)}$ & 2001 & 68 & M & C7-T1 & P & B & No OR & $\mathrm{E}$ & $10 \mathrm{~h}$ & HTN \\
\hline Hentschel $^{25)}$ & 2001 & 65 & $\mathrm{~F}$ & T7-T12 & A & A & No OR & $\mathrm{E}$ & $20 \mathrm{~min}$ & HTN \\
\hline Kim & 2002 & 78 & $\mathrm{~F}$ & T10-L4 & $P$ & C & 16 & D & hrs & HTN, DM \\
\hline Kim & 2002 & 71 & $M$ & C2-T4 & $P L$ & C & $<24$ & $E$ & hrs & syncope \\
\hline Steinmetz ${ }^{26)}$ & 2003 & 27 & $\mathrm{~F}$ & $\mathrm{~T} 1-\mathrm{T} 2$ & A & C & $<24$ & $\mathrm{E}$ & lwk & pregnant \\
\hline Rosenberg $^{27)}$ & 2003 & 14 & $\mathrm{~F}$ & T3-T5 & $\mathrm{PL}$ & C & $<24$ & $\mathrm{E}$ & $\min$ & None \\
\hline Kong $^{28)}$ & 2003 & 52 & $\mathrm{~F}$ & T8-T10 & $P$ & D & 15 & $\mathrm{E}$ & $\min$ & Renal failure \\
\hline Muizelaar & 2003 & 42 & $\mathrm{~F}$ & Т9-Т12 & $\mathrm{P}$ & A & $<24$ & A & hrs & Aspirin \\
\hline Kong $^{28)}$ & 2003 & 77 & $\mathrm{~F}$ & C7-Т3 & $P$ & A & 34 & A & $2 \mathrm{~h}$ & HTN \\
\hline
\end{tabular}


Table 2. Demographics of SSEH patients (continue)

\begin{tabular}{|c|c|c|c|c|c|c|c|c|c|c|}
\hline Author & Year & Age & Gender & Level & Position & $\begin{array}{c}\text { Initial } \\
\text { Frankel }\end{array}$ & $\begin{array}{c}\text { Time to } \\
\text { Operation (hrs) }\end{array}$ & $\begin{array}{c}\text { Final } \\
\text { Frankel }\end{array}$ & $\begin{array}{c}\text { Duration of } \\
\text { Symptoms }\end{array}$ & $\mathrm{PMH}$ \\
\hline Muthukumar $^{29)}$ & 2003 & 19 & $M$ & C2-C7 & $A$ & C & No OR & $E$ & $30 d$ & None \\
\hline Kirazli $i^{30)}$ & 2004 & 22 & M & T9-T10 & $P$ & $A$ & 10 & $E$ & $4 d$ & AntiCoag \\
\hline Masski $^{31)}$ & 2004 & 27 & $\mathrm{~F}$ & C7-T2 & $A$ & $A$ & 12 & $A$ & $5 d$ & Pregnant \\
\hline Cywinsky $^{32)}$ & 2004 & 27 & $\mathrm{~F}$ & $\mathrm{~T} 1-\mathrm{T} 2$ & $A$ & C & 36 & $E$ & $10 d$ & Pregnant \\
\hline $\mathrm{Jea}^{33)}$ & 2005 & 24 & $\mathrm{~F}$ & $\mathrm{~T} 1-\mathrm{T} 2$ & PL & $A$ & 8 & $E$ & $2 w k$ & pregnant \\
\hline Song ${ }^{34)}$ & 2005 & 51 & M & T8-L2 & $P L$ & $A$ & 28 & A & hrs & Ml, Aspirin \\
\hline Hsieh $^{35)}$ & 2006 & 75 & $\mathrm{~F}$ & $\mathrm{C} 2-\mathrm{C} 5$ & $\mathrm{PL}$ & $A$ & $>4$ & D & Unk & $\mathrm{HTN}$ \\
\hline Hsieh $^{35)}$ & 2006 & 20 & M & C5-C7 & PL & B & $>8$ & $E$ & Unk & None \\
\hline Hsieh $^{35)}$ & 2006 & 62 & $\mathrm{~F}$ & C3-C5 & PL & C & No OR & C & $2 w k$ & Unk \\
\hline Hsieh $^{35)}$ & 2006 & 54 & M & C3-C5 & PL & B & $6 \mathrm{~h}$ & $E$ & Unk & Type I| Diabetes \\
\hline Villas ${ }^{36)}$ & 2006 & 64 & M & C4-C5 & $P L$ & B & Unk & $E$ & unk & None \\
\hline $\mathrm{Pai}^{37)}$ & 2006 & 1.25 & M & C4-T3 & $A$ & C & 48 & $E$ & Unk & Unk \\
\hline Shin $^{38)}$ & 2007 & 16 & $\mathrm{~F}$ & C7-T2 & A & B & $<24$ & C & Unk & None \\
\hline Abram ${ }^{39}$ & 2007 & 10 & $\mathrm{~F}$ & C7-T1 & $P$ & B & 42 & B & 42 & None \\
\hline Park $^{40)}$ & 2007 & 68 & $\mathrm{~F}$ & T7-T12 & $P$ & $A$ & 12 & A & Unk & None \\
\hline Helmy ${ }^{41)}$ & 2007 & 65 & $\mathrm{~F}$ & C3-C4 & $P$ & $A$ & $<24$ & C & Unk & None \\
\hline $\operatorname{Lee}^{42)}$ & 2007 & 0.33 & M & C4-T4 & $P L$ & C & $>48$ & $E$ & Unk & None \\
\hline Poonai ${ }^{43)}$ & 2007 & 0.92 & $\mathrm{~F}$ & C4-T3 & $\mathrm{P}$ & C & $>48$ & D & Months & None \\
\hline Kalina $^{44)}$ & 2007 & 0.58 & M & C2-L4 & $\mathrm{P}$ & C & No OR & $E$ & Days & Severe Hemophilia \\
\hline Bose ${ }^{45)}$ & 2007 & 24 & $\mathrm{~F}$ & $\mathrm{C} 4-\mathrm{C} 7$ & $P$ & $A$ & $>48$ & A & Unk & Post-Partum \\
\hline $\operatorname{Riaz}^{46)}$ & 2007 & 52 & M & C6-T1 & $P$ & C & $>36$ & $E$ & Unk & HTN, Smoker \\
\hline Bisson $^{47)}$ & 2007 & 7 & M & C2-T3 & $\mathrm{P}$ & $\mathrm{D}$ & No OR & $\mathrm{E}$ & 5 days & Hemophilia B \\
\hline Fok $^{48)}$ & 2007 & 1.25 & M & С3-Т8 & $\mathrm{P}$ & $\mathrm{B}$ & $>48$ & $\mathrm{E}$ & 2 days & None \\
\hline Sakakibara $^{49)}$ & 2008 & 55 & M & $\mathrm{C} 2-\mathrm{C} 7$ & $A$ & B & Unk & $\mathrm{D}$ & Unk & Unk \\
\hline Ramelli ${ }^{50)}$ & 2008 & 13.5 & M & $\mathrm{C6}-\mathrm{C} 7$ & $P$ & $A$ & Unk & D & Unk & Unk \\
\hline Ramelli ${ }^{50)}$ & 2008 & 0.58 & $\mathrm{~F}$ & C6-T7 & $P$ & $A$ & $>36$ & $A$ & Unk & None \\
\hline $\operatorname{Liv}^{51)}$ & 2008 & 50 & M & T6-T8 & P & B & Unk & $\mathrm{D}$ & Unk & None \\
\hline Thiele ${ }^{52)}$ & 2008 & 55 & $\mathrm{~F}$ & $\mathrm{C} 4-\mathrm{C} 7$ & $P L$ & D & Unk & $\mathrm{E}$ & Unk & None \\
\hline Morales Ciancio ${ }^{53)}$ & 2008 & 78 & $\mathrm{~F}$ & T1-T12 & $P$ & C & $>48$ & C & $>48$ & Clopidogrel \\
\hline Vitali ${ }^{54)}$ & 2008 & 15 & M & $\mathrm{C} 5-\mathrm{C} 6$ & $\mathrm{~L}$ & C & No OR & $E$ & Days & None \\
\hline Halim $^{55}$ & 2008 & 25 & M & $\mathrm{C} 2-\mathrm{T} 1$ & $\mathrm{P}$ & $A$ & No OR & $\mathrm{D}$ & 10 days & None \\
\hline Aksay ${ }^{56)}$ & 2008 & 32 & M & C7-T3 & $\mathrm{P}$ & $A$ & $<24$ & D & 1 week & None \\
\hline Baek $^{57)}$ & 2008 & 69 & $\mathrm{~F}$ & C2-C5 & $\mathrm{L}$ & C & $>2$ & D & 1 week & None \\
\hline Baek $^{57)}$ & 2008 & 64 & M & T5-L2 & L & C & $>2$ & $\mathrm{E}$ & Unk & None \\
\hline Baek $^{57)}$ & 2008 & 19 & M & C3-C7 & $P L$ & C & $>4$ & $E$ & Unk & None \\
\hline $\operatorname{Lim}^{58)}$ & 2008 & 1.66 & $\mathrm{~F}$ & C7-T4 & $P$ & C & $>48$ & $\mathrm{D}$ & 2 weeks & None \\
\hline$O h^{59)}$ & 2008 & 62 & M & T9-T12 & $P$ & $A$ & 10 & $E$ & 10 & HTN \\
\hline Hussenbocus $^{60)}$ & 2009 & 36 & M & C7-T4 & $P$ & A & $<12$ & A & 5 & None \\
\hline Forsnes $^{61)}$ & 2009 & 32 & $\mathrm{~F}$ & T12-L2 & $\mathrm{P}$ & C & 16 & $\mathrm{D}$ & 2 days & Pregnant \\
\hline Nojiri ${ }^{62)}$ & 2009 & 55 & M & C5-T3 & $\mathrm{L}$ & B & $>24$ & $\mathrm{D}$ & $>24$ & None \\
\hline Nourbakshs $^{63)}$ & 2009 & 75 & M & C3-T2 & $L$ & C & 50 & $\mathrm{D}$ & 3 months & None \\
\hline Kunizawa ${ }^{64)}$ & 2009 & 63 & $\mathrm{~F}$ & C3-T5 & $P L$ & $A$ & 7 & $\mathrm{D}$ & 6 months & None \\
\hline Woon $^{65)}$ & 2009 & 33 & $\mathrm{FF}$ & T2-T4 & $P$ & $A$ & $14 \mathrm{~h}$ & $\mathrm{E}$ & $8 h$ & HTN \\
\hline Aoki ${ }^{66)}$ & 2009 & 82 & $\mathrm{~F}$ & T10-T12 & $P$ & C & 8 & $D$ & 2 days & Anticoag \\
\hline Aoki ${ }^{66)}$ & 2009 & 64 & M & C2-T3 & $\mathrm{P}$ & B & No OR & $\mathrm{E}$ & 5 & Anticoag \\
\hline Chen $^{67)}$ & 2009 & 25 & M & C6-T1 & $P$ & B & 16 & $E$ & 36 & None \\
\hline Deger $^{68)}$ & 2009 & 77 & $\mathrm{~F}$ & C2-T9 & $\mathrm{P}$ & B & 4 & C & Unk & HTN, renal failure \\
\hline Muizelaar & 2003 & 42 & $\mathrm{~F}$ & T9-T12 & $P$ & $A$ & $<24$ & $A$ & hrs & Aspirin \\
\hline Kong $^{28)}$ & 2003 & 77 & $\mathrm{~F}$ & C7-T3 & $\mathrm{P}$ & A & 34 & A & $2 \mathrm{~h}$ & HTN \\
\hline
\end{tabular}


Table 2. Demographics of SSEH patients (continue)

\begin{tabular}{|c|c|c|c|c|c|c|c|c|c|c|}
\hline Author & Year & Age & Gender & Level & Position & $\begin{array}{c}\text { Initial } \\
\text { Frankel }\end{array}$ & $\begin{array}{c}\text { Time to } \\
\text { Operation (hrs) }\end{array}$ & $\begin{array}{c}\text { Final } \\
\text { Frankel }\end{array}$ & $\begin{array}{l}\text { Duration of } \\
\text { Symptoms }\end{array}$ & $\mathrm{PMH}$ \\
\hline Miller $^{69)}$ & 2010 & 58 & $M$ & T8-L2 & $P$ & $A$ & $>48$ & $A$ & 4 days & HTN, diabetes \\
\hline Tagaya $^{70)}$ & 2010 & 0.33 & $M$ & C2-L2 & $P$ & C & No OR & $E$ & Unk & Hemophilia \\
\hline Subbiah $^{71)}$ & 2010 & 65 & $M$ & $\mathrm{C} 4-\mathrm{T} 1$ & $A$ & C & No OR & $E$ & 24 hours & LMWHT \\
\hline Kiehna $^{72)}$ & 2010 & 0.42 & $M$ & Cl-L4 & $P$ & $\mathrm{D}$ & No OR & $E$ & 4 hours & Hemophilia \\
\hline Hosoki $^{73)}$ & 2010 & 0.92 & M & T9-L1 & $P$ & $\mathrm{D}$ & 48 & $E$ & 2 days & None \\
\hline Kumchev ${ }^{74)}$ & 2010 & 55 & $M$ & $\mathrm{C} 2-\mathrm{C} 4$ & $P L$ & B & $<24$ & $E$ & $\mathrm{Hrs}$ & None \\
\hline $\operatorname{Sirin}^{75)}$ & 2010 & 77 & M & C6-T3 & $P$ & C & No OR & $\mathrm{E}$ & 40 minutes & HTN \\
\hline Fleager $^{76)}$ & 2011 & 22 & $M$ & C2-L3 & $A$ & $A$ & 4 & $E$ & 2 days & None \\
\hline Badar $^{77)}$ & 2011 & 35 & $\mathrm{~F}$ & Т2-Т8 & $A$ & C & $>24$ & $E$ & 3 days & None \\
\hline Matsubara $^{78)}$ & 2011 & 36 & $\mathrm{~F}$ & C3-C7 & $P$ & $B$ & $9 \mathrm{~h}$ & C & $9 \mathrm{~h}$ & None \\
\hline Chang $^{79)}$ & 2011 & 32 & $\mathrm{~F}$ & C3-C7 & $P$ & $\mathrm{~B}$ & $<12$ & C & Unk & Renal failure \\
\hline
\end{tabular}

Table 3. Number of patients who presented with varying degrees of neurological dysfunction, as described by the Frankel grade

\begin{tabular}{cc}
\hline \hline Frankel Grade at Presentation: & Number of Cases: \\
\hline A & $39 / 107(36 \%)$ \\
B & $16 / 107(15 \%)$ \\
C & $38 / 107(36 \%)$ \\
D & $11 / 107(10 \%)$ \\
E & $3 / 107(3 \%)$ \\
\hline
\end{tabular}

involved was 5.4 with a range of 1 to 23 . The distribution of segments showed increased involvement at the cervicothoracic junction (Fig. 6). The majority (53\%) of hematomas were located posterior to the spinal cord, whereas $23 \%$ were located posterolateral, $18 \%$ were anterior, $5 \%$ were lateral and $1 \%$ were circumferential to the cord.

Overall, $60 / 107(56 \%)$ of the patients had complete recovery. Of the 80 operative cases, 35 (43.75\%) experienced complete recovery, $22(27.5 \%)$ had incomplete motor recovery with good functional outcome, 10 (12.5\%) had incomplete motor recovery with poor functional outcome, 2 (2.5\%) had preserved sensation without motor function and 11 (13.75\%) had no motor or sensory recovery. No patients suffered from worse neurological outcome after treatment.

For the statistical analysis investigating the effect of surgical timings on patient outcome, 43 of the 107 patients were excluded from the analysis for the following reasons: twenty-three patients were managed conservatively and thus would not provide any information on the effect of delayed surgery. We also excluded nine infants ( $<2$ years old) from the analysis due to considerable delays in diagnosis, and consequently, substantially late presentation to the OR. As a result, age and time of surgery would be confounded. Finally, for six patients, missing data precluded analysis.

Of the 64 patients analyzed, 31 (48\%) were female and 33
$(52 \%)$ were male. The mean age of the patients was $48( \pm 24)$ with the following distribution among the four age categories: less than 20 years $(n=9), 20$ to 40 years $(n=16), 40$ to 60 years $(n=12)$ and over 60 years $(n=27)$. Forty-nine patients had surgery within 24 hours and 19 patients had surgery after 24 hours. The number of patients with an initial Frankel grade of $\mathrm{A}, \mathrm{B}, \mathrm{C}$ and $\mathrm{D}$ were 28, 9, 22 and 5, respectively.

Initial Frankel grade $(F(1,60)=23.15, p=0.001)$ and sex $(\mathrm{F}(1,60)=4.41, \mathrm{p}=0.040)$ were statistically significant to final Frankel grade. For every one grade increase in the initial grade, the final grade increased by 0.69 . After controll- ing for initial Frankel grade, male patients had better outcomes from surgery than female patients. The final Frankel grade of male patients averaged 0.63 points higher than female patients after adjusting for initial Frankel grade and time to surgery.

Although the timing of the surgery after admission was not statistically significant $(\mathrm{F}(1,60)=3.18, \mathrm{p}=0.079)$ to final Frankel grade, early surgery (within 24 hours) yielded better outcomes than delayed surgery (after 24 hours) (Fig. 7). On average, patients who received surgery within 24 hours had a final Frankel grade 0.60 points higher than those receiving later surgery (Fig. 8).

On the other hand, age $(\mathrm{F}(3,57)=0.96, \mathrm{p}=0.42)$, past history of hypertension $(\mathrm{F}(1,57)=0.0013, \mathrm{p}=0.97)$, use of anticoagulants $(\mathrm{F}(1,57)=0.57, \mathrm{p}=0.45)$, and the number of spinal segments in the hematoma $(\mathrm{F}(1,57)=0.99, \mathrm{p}=0.32)$ were not significant predictors of final Frankel grade. In addition, no two-way interactions were significant.

\section{DISCUSSION}

The first reported case of SSEH was by Jackson in 1869 . Since then, over 400 cases have been described in the world literature. MRI has emerged as the diagnostic study of choice 


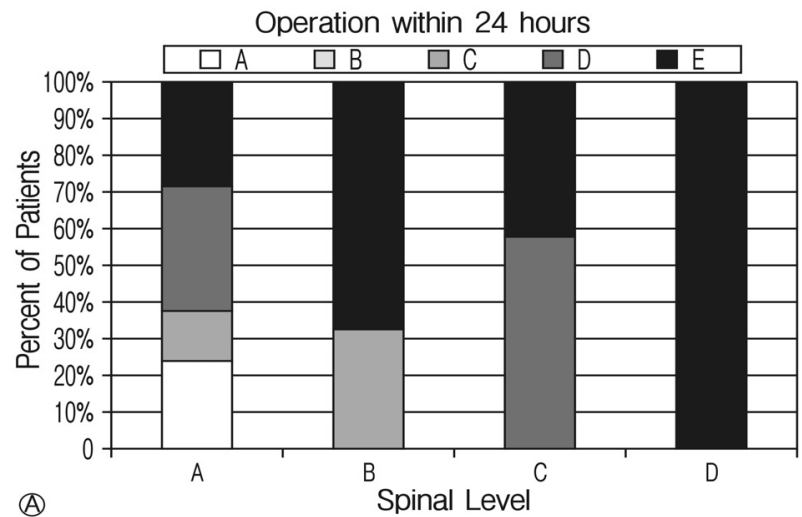

(A)

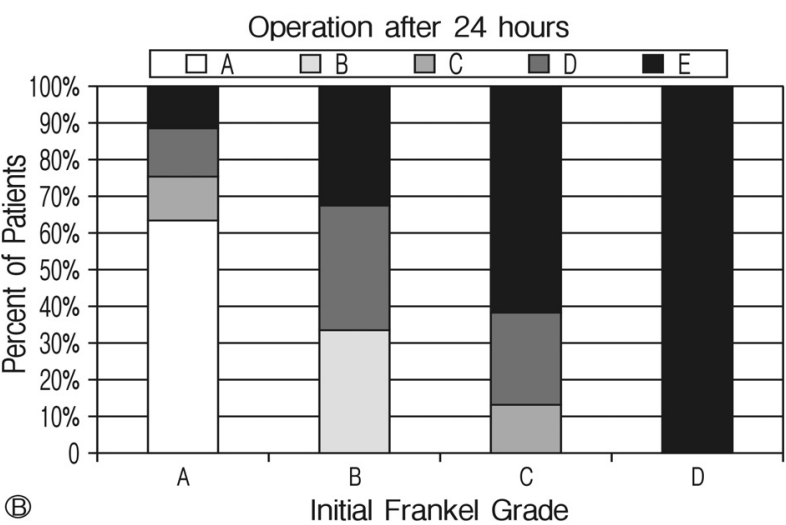

Fig. 7. Approximately $62 \%$ patients with an initial Frankel grade of $A$ that had surgery after 24 hours do not improve. In contrast, only about $24 \%$ of Initial A; grade patients operated within 24 hours failed to improve. All patients with an initial Frankel grade of $B$; improved when operated on within 24 hours while $33 \%$ of those operated on after 24 hours remained without motor function.

for SSEH. A T2-weighted scan showing an epidural mass hyperintense to the spinal cord with focal areas of hypointensity (consistent with the presence of deoxyhemoglobin) highly suggests the presence of an epidural hematoma ${ }^{26,27}$. No MRI characteristic that may predict outcome currently exists ${ }^{27}$.

In the majority of reports, the hematoma is considered to be "spontaneous" if it develops without known cause (i.e. trauma, fracture, spinal lesion or medical procedure). Hence, some refer to SSEH as idiopathic spinal epidural hematoma ${ }^{41)}$. In most cases, however, patients have some history (i.e. anticoagulation use, bleeding diathesis, hypertension, straining) that may contribute to the bleeding. Some case reports include SSEH due to vascular malformations, whereas most authors do not consider these to be spontaneous in origin. Following a variable time interval anywhere from minutes to weeks, neurological dysfunction develops ranging from mild weakness to complete paralysis. Past reviews found that better prognosis is associated with less severe initial neurological dysfunction and with

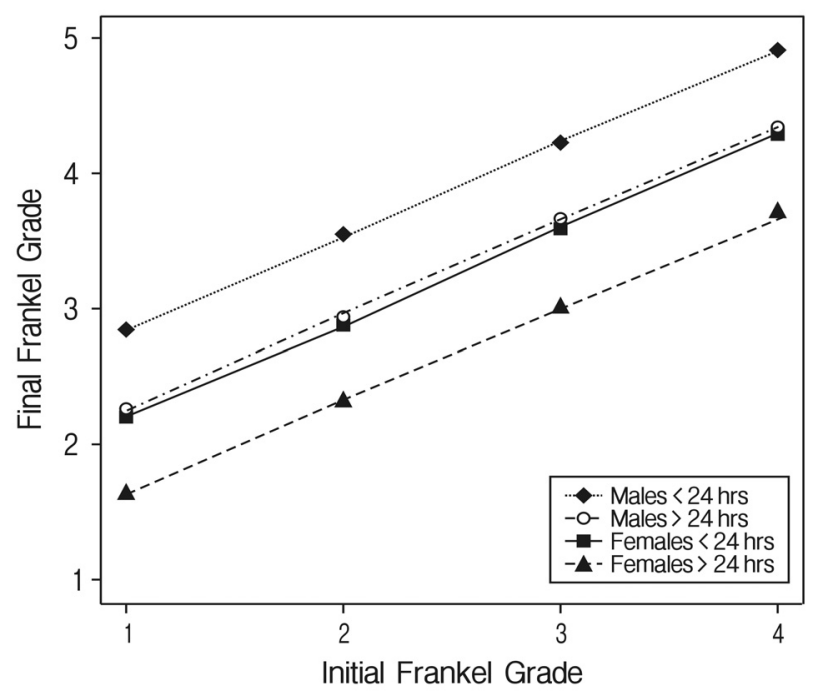

Fig. 8. Initial and final Frankel grades.

faster time to operation ${ }^{30,36,41,50)}$. However, reported cases of SSEH managed non-surgically with steroids ${ }^{21)}$ or only observation also exist ${ }^{17}$.

Past reviews found that pediatric patients presented with longer and more variable times to neurological symptom onset ${ }^{17}$. We believe this is due to difficulty in assessing pain and performing accurate neurologic examination in this group. Children under two years of age presented to surgeons after days, and sometimes weeks after symptoms eventually alerted parents or caregivers. Such symptoms included chronic irritability, crying and inability to ambulate or stand. Elderly patients, on the other hand, had faster onset to deficits varying from minutes to hours.

Treatment consisted of either decompressive laminectomy or conservative management with or without steroids. In our study, conservative management was employed in 23 patients. In one of these, neurological improvement occurred following a lumbar puncture (LP), which drew $20 \mathrm{ml}$ of clear cerebrospinal fluid $(\mathrm{CSF})^{3)}$. In this case, the LP was performed to rule out infection before MRI diagnosis of the epidural hematoma. The remaining 84 patients were treated surgically. Of these, 47 were operated within 24 hours of symptom onset and 37 were operated 24 hours after symptom onset.

\section{Prognostic Factors}

For those cases treated surgically, the literature reports that post-operative recovery is based both on pre-operative neurological function as well as time to operation after the onset of these symptoms ${ }^{30,41,50)}$. Our review also illustrates that patients with a higher pre-operative Frankel grade, functional recovery 
was more likely. Also, those patients with a shorter interval to operation had better recovery than those with longer intervals. Especially in cases of complete neurological dysfunction, a timely operation may be of great benefit, and even in cases of delayed surgery, good functional outcome can be achieved. In contrast to Groen's review and for unknown reason, recovery was greater in males than in females after surgery.

A past medical history that may have contributed to bleeding is associated with a worse neurological presentation and may indirectly influence eventual recovery. In 10 of the 26 cases of complete initial motor deficit, patients had a history of anticoagulation such as aspirin or ibuprofen use.

However, even patients with significant comorbidities did achieve functional recovery comparable with patients without comorbidities. Hence, a relevant past medical history does not predict poor outcome, but may be associated with worse initial neurological dysfunction than others.

Initial neurological dysfunction was the strongest predictor for a patient's outcome. Patients presenting with severe and/or worsening conditions typically did not recover as well as those presenting with minor symptoms. Furthermore, the timing of a decompressive laminectomy was more significant factor in patients initially presenting with Frankel grades of A or B. Patients presenting with better neurologic function (scores of D or E) frequently showed substantial improvement regardless of time to operation. With the exception of patients showing significant neurologic improvement before surgery, a prompt evacuation of an epidural hematoma should be attempted. Even in the case of complete or severe neurologic deficit and lengthy symptom duration, functional recovery may be achieved through surgical intervention.

\section{Pathophysiology}

Groen speculated that bleeding most likely arises from the posterior epidural venous plexus ${ }^{33)}$. This plexus lies directly between the dura and ligamentum flavum making rupture more likely than in the anterior venous plexus which is covered by the posterior longitudinal ligament. Communication between the venous plexus and the intrathoracic veins allows for transfer of pressures from one compartment to another. Given that the epidural venous plexus is devoid of valves, it may be particularly vulnerable to sudden changes in pressure, especially at the cervicothoracic and thoracolumbar junctions where resistance is minimal. Cases of SSEH associated with heavy lifting, straining, pregnancy and hypertension further supports the venous theory.

In contrast, Beatty suggested that bleeding occurs from epidural arteries ${ }^{16}$. He argued that hemorrhage from an epidural vein would not create enough pressure to compress the spinal cord, observing that the normally expanded epidural sac may tamponade epidural bleeding encountered during surgical procedures. There are also reports of SSEH located anteriorly for which the posterior venous plexus theory would not apply $4,5,11,12,19,20,24,26,28,29,34,37,44,51)$. With few exceptions, SSEH arises from a venous bleed rather than an arterial bleed.

\section{Treatment}

In recent years, there have been increased reports of spontaneously resolving spinal epidural hematomas. This may be due to quick diagnosis by higher resolution MRI when a patient presents with sudden onset of neurological deficits. Even in the face of improving neurological condition, MRI is performed to evaluate the size and location of the hematoma ${ }^{32}$. It is important to stress that by the time of diagnosis with MRI, the patients managed conservatively were already improving clinically. Thus, conservative management is only appropriate in the face of improving neurological status.

Four of the five hemophiliac patients in our review were treated conservatively with either Factor VIII or Factor IX? both of which are clotting-related proteins. All four of these patients recovered to an "E" Frankel grade. Although the number of hemophiliac patients is relatively small, there has been sub stantial success of conservative management in these patients.

In a review by Groen, conservatively treated SSEH had more spinal levels involved than those treated operatively. Similarly in our review, the mean spinal levels of SSEH managed conservatively was seven as opposed to five for surgical cases. The total length of involvement was not a dependent factor predicting outcome.

\section{CONCLUSION}

Both the venous and arterial theories provide plausible explanations for SSEH with most cases probably resulting from a venous bleed. Our review of SSEH presented in the English literature since 1996 is consistent with previously published reviews with regard to clinical presentation, spinal segment involvement and factors predicting neurological outcome except males as a group did better than females for unknown reason. Patients with less initial neurological deficit and those with shorter time to operation show better neurological improvement. Those who present with a past medical history are more likely to have greater initial neurological deficit with tendency to greater bleeding, especially in the case of anticoagulation use. An increasing number of conservatively treated SSEH cases have been reported. In all such cases, patients presented with improving neurological status. Although high resolu- 
tion MRI has improved the accuracy of SSEH diagnosis, no imaging characteristics serve to predict the eventual outcome. Expedient decompressive laminectomy continues to be the treatment of choice for patients with SSEH.

\section{Acknowledgments}

We would like to acknowledge Sandra Taylor, $\mathrm{PhD}$ for her guidance and feedback in statistical analysis. Statistical support was made possible by grant \# UL1 RR024146 from the National Center for Research Resources (NCRR), a component of the National Institute of Health (NIH) and NIH Roadmap for Medical Research. The contents are solely the responsibility of the authors and do not necessarily represent the official view of NCRR or NIH.

\section{REFERENCES}

1. Abram HS, DeLaHunt MJ, Merinbaum DJ, Hammond DN: Recurrent spontaneous spinal epidural hematoma in a child: first case report. Pediatr Neurol 36(3):177-180, 2007

2. Alexiadou-Rudolf C, Ernestus RI, Nanassis K, Lanfermann H, Klug N: Acute nontraumatic spinal epidural hematomas. An important differential diagnosis in spinal emergencies. Spine 23(16):1810-1813, 1998

3. Alexiadou-Rudolf C, Ernestus RI, Nanassis K, Lanfermann H, Klug N: Acute nontraumatic spinal epidural hematomas. An important differential diagnosis in spinal emergencies. Spine 23(16):1810-1813, 1998

4. Aoki Y, Yamagata M, Shimizu K, Ikeda Y, Nakajima F, Ohtori $\mathrm{S}$, et al: An unusually rapid spontaneous recovery in a patient with spinal epidural hematoma. J Emerg Med 2009

5. Awada A, Russell N, al Fayez N, Naufal R, al Kohlani H: Spontaneous cervical epidural hematoma: case report. Spinal Cord 36:71-72, 1998

6. Badar F, Kirmani S, Rashid M, Azfar SF, Yasmeen S, Ullah E: Spontaneous spinal epidural hematoma during pregnancy: a rare obstetric emergency. Emerg Radiol 18(5):433-436, 2011

7. Baek BS, Hur JW, Kwon KY, Lee HK: Spontaneous spinal epidural hematoma. J Korean Neurosurg Soc 44(1):40-42, 2008

8. Bisson EF, Dumont T, Tranmer B: Spontaneous spinal epidural hematoma in a child with hemophilia B. Can J Neurol Sci 34(4):488-490, 2007

9. Bose S, Ali Z, Rath GP, Prabhakar H: Spontaneous spinal epidural haematoma: a rare cause of quadriplegia in the post-partum period. Br J Anaesth 99(6):855-857, 2007

10. Carroll SG, Malhotra R, Eustace D, Sharr M, Morcos S: Spontaneous spinal extradural hematoma during pregnancy. J Mater Fetal Med 6:218-219, 1997

11. Chang MO, Koh ES, Kim MJ, Chang YS, Chung S: Spontaneous spinal epidural hematoma. QJM, 2011

12. Chen CJ, Fang W, Chen CM, Wan YL: Spontaneous spinal epidural hematomas with repeated remission and relapse. Neuroradiology 39:737-740, 1997

13. Chen CL, Lu CH, Chen NF: Spontaneous spinal epidural hematoma presenting with quadriplegia after sit-ups exercise. Am J Emerg Med 27(9):1170 e3-7, 2009

14. Cirak B, Guven MB, Akalan N: Spontaneous spinal epidural hematoma. Arch Phys Med Rehabil 80(1):125, 1999

15. Cywinski JB, Parker BM, Lozada LJ: Spontaneous spinal epidural hematoma in a pregnant patient. J Clin Anesth 16:371375, 2004

16. David S, Salluzzo RF, Bartfield JM, Dickinson ET: Spontaneous cervicothoracic epidural hematoma following prolonged valsalva secondary to trumpet playing. Am J Emerg Med 15: 73-75, 1997

17. Deger SM, Emmez H, Bahadirli K, Kale A, Ebinc FA, Turkoglu $\mathrm{M}$, et al: A spontaneous spinal epidural hematoma in a hemodialysis patient: a rare entity. Intern Med 48(24):2115-2118, 2009

18. Duffill J, Sparrow OC, Millar J, Barker CS: Can spontanoeous spinal epidural hematoma be managed safely without operation? a report of four cases. J Neurol Neurosurg Psychiatry 69:816-819, 2000

19. Forsnes E, Occhino A, Acosta R: Spontaneous spinal epidural hematoma in pregnancy associated with using low molecular weight heparin. Obstet Gynecol 113:532-533, 2009

20. Fukui MB, Swarnkar AS, Williams RL: Acute spontaneous spinal epidural hematomas. AJNR 20:1365-1372, 1999

21. Gaffney P, Guthrie JA: Spontaneous spinal epidural haematoma: an unusual cause of neck pain. J Accid Emerg Med 17: 229-232, 2000

22. Groen RJ, van Alphen HA: Operative treatment of spontaneous spinal epidural hematomas: A study of the factors predicting postoperative outcome. Neurosurgery 39(3):494-509, 1996

23. Groen RJ: Non-operative treatment of spontaneous spinal epidural hematomas: a review of the literature and a comparison with operative cases. Acta Neurochir 146:103-110, 2004

24. Halim TA, Nigam V, Tandon V, Chhabra HS: Spontaneous cervical epidural hematoma: report of a case managed conservatively. Indian J Orthop 42(3):357-359, 2008

25. Helmy A, Mellor G: Spontaneous cervical cord haemorrhage: an unusual presentation. Emerg Med J 24(3):e16, 2007

26. Hentschel SJ, Woolfenden AR, Fairholm DJ: Resolution of spontaneous spinal epidural hematoma without surgery: report of two cases. Spine 26(22):E525-527, 2001

27. Hsieh CT, Chang CF, Lin EY, Tsai TH, Chiang YH, Ju DT: Spontaneous spinal epidural hematomas of cervical spine: report of 4 cases and literature review. Am J Emerg Med 24 (6):736-740, 2005

28. Inamasu J, Hori S, Aoki K, Aikawa N, Maruiwa H, Toyama Y: Spontaneous spinal epidural hematoma. Am J Emerg Med 18(7):837-839, 2000

29. Jea A, Moza K, Levi AD, Vanni S: Spontaneous spinal epidural hematoma during pregnancy: case report and literature review. Neurosurgery 56(5):E1156, 2005

30. Kiehna EN, Waldron PE, Jane JA: Conservative management 
of an acute spontaneous holocord epidural hemorrhage in a hemophiliac infant. J Neurosurg Pediatr 6(1):43-48, 2010

31. Kiehna EN, Waldron PE, Jane JA: Conservative management of an acute spontaneous holocord epidural hemorrhage in a hemophiliac infant. J Neurosurg Pediatr 6(1):43-48, 2010

32. Kirazli Y, Akkoc Y, Kanyilmaz S: Spinal epidural hematoma associated with oral anticoagulation therapy. Am J Phys Med Rehabil 83(3):220-223, 2004

33. Kong J, Mak KH: Spontaneous spinal epidural haematoma - An unusual cause of spinal cord compression. Hong Kong Med J 9(1):55-57, 2003

34. Kotilainen EM, Pajulo O: Spontaneous epidural hematoma as a cause of sciatic pain in a schoolboy. Pediatr Neurol 17(4):350352, 1997

35. Lawton MT, Porter RW, Heiserman JE, Jacobowitz R, Sonntag VK, Dickman CA: Surgical management of spinal epidural hematoma: relationship between timing and neurological outcome. J Neurosurg 83(1):1-7, 1995

36. Liu WH, Hsieh CT, Chiang YH, Chen GJ: Spontaneous spinal epidural hematoma of thoracic spine: a rare case report and review of literature. Am J Emerg Med 26(3):384.e1-2, 2008

37. Lonjon MM, Paquis P, Chanalet S, Grellier P: Nontraumatic spinal epidural hematoma: report of four cases and review of the literature. Neurosurgery 41(2):483-487, 1997

38. Meena AK, Jayalakshmi S, Prasad VS, Murthy JM: Spinal epidural haematoma in a patient with haemophilia-B. Spinal Cord 36:658-660, 1998

39. Miller JB, Khalsa G, Vohra T: Spontaneous spinal epidural hematoma presenting as flank pain and constipation. Am J Emerg Med 28(4):536 e3-5, 2010

40. Morales Ciancio RA, Drain O, Rillardon L, Guigui P: Acute spontaneous spinal epidural hematoma: an important differential diagnosis in patients under clopidogrel therapy. Spine J 8(3):544-547, 2008

41. Muthukumar N: Chronic spontaneous spinal epidural hematoma - a rare cause of cervical myelopathy. Eur Spine J 12: 100-103, 2003

42. Nourbakhsh A, Chaljub G, Garges KJ: Spontaneous cervical epidural hematoma masquerading as an abscess on magnetic resonance imaging scan. J Manipulative Physiol Ther 32(5): 391-395, 2009

43. Oh JY, Lingaraj K, Rahmat R: Spontaneous spinal epidural haematoma associated with aspirin intake. Singapore Med J 49(12):e353-355, 2008

44. Pahapill PA, Lownie SP: Conservative treatment of acute spontaneous spinal epidural hematoma. Can J Neurol Sci 25:159163, 1998

45. Pai SB, Maiya PP: Spontaneous spinal epidural hematoma in a toddler - a case report. Childs Nerv Syst 22(5):526-529, 2006

46. Poonai N, Rieder MJ, Ranger A: Spontaneous spinal epidural hematoma in an 11-month-old girl. Pediatr Neurosurg 43(2): 121-124, 2007

47. Ramelli GP, Boscherini D, Kehrli P, Rilliet B: Spontaneous spinal epidural hematomas in children: can we prevent a negative prognosis? - - reflections on 2 cases. J Child Neurol 23 (5):564-567, 2008

48. Rohde V, K?ker W, Reinges MH, Gilsbach JM: Microsurgical treatment of spontaneous and non-spontaneous spinal epidural haematomas: neurological outcome in retalion of aetiology. Acta Neurochir 142:787-793, 2000

49. Rosenberg O, Itshayek E, Israel Z: Sontaneous spinal epidural hematoma in a 14 year old girl. Pediatr Neurosurg 38:216218, 2003

50. Sakakibara R, Yamazaki M, Mannouji C, Yamaguchi C, Uchiyama $\mathrm{T}$, Ito $\mathrm{T}$, et al: Urinary retention without tetraparesis as a sequel to spontaneous spinal epidural hematoma. Intern Med 47(7):655-657, 2008

51. Sirin S, Arslan E, Yasar S, Kahraman S: Is spontaneous spinal epidural hematoma in elderly patients an emergency surgical case? Turk Neurosurg 20(4):557-560, 2010

52. Song KJ, Lee KB: The poor ouctome of the delayed diagnosis of acute spontaneous spinal epidural hematoma: two cases report. J Korean Med Sci 20:331-334, 2005

53. Van Schaeybroeck P, Van Calenbergh F, Van De Werf F, Demaerel P, Goffin J, Plets C: Spontaneous spinal epidural hematoma associated with thrombolysis and anticoagulation therapy: report of three cases. Clin Neurol Neurosurg 100: 283-287, 1998

54. Wagner S, Forsting M, Hacke W: Spontaneous resolution of a large spinal epidural hematoma: case report. Neurosurgery 38:816-818, 1996

55. Woon CY, Peng BC, Chen JL: Spontaneous spinal epidural haematomas and the prognostic implications of interval to surgical decompression: a report of two cases. J Orthop Surg (Hong Kong) 17(2):216-219, 2009 